\title{
Analysis of a MAC Protocol for a Time-Code Air Interface in LEO Mobile Satellite Systems
}

\author{
Romano Fantacci ${ }^{1}$ and Giovanni Giambene ${ }^{2}$
}

1 Dipartimento di Ingegneria Elettronica e Telecomunicazioni - Università degli Studi di Firenze, Via S. Marta, 3 - 50139 Firenze, ITALY,

fantacci@lenst.det.unifi.it,

2 Dipartimento di Ingegneria dell'Informazione - Università degli Studi di Siena, Via Roma, 56 - 53100 Siena, ITALY, giambene@unisi.it

\begin{abstract}
This paper deals with Low Earth Orbit - Mobile Satellite Systems (LEO-MSSs) and proposes a novel Medium Access Control (MAC) scheme for a hybrid time-code wideband air interface. This is a reservation scheme where each mobile terminal that needs to transmit makes random accesses (on available time-code resources) until it receives a positive acknowledgment from the satellite. Our protocol is named Code Division-Packet Reservation Multiple Access scheme with Hindering States. This work has been carried out within the "Multimedialità" Project of the Italian National Consortium for Telecommunications.
\end{abstract}

Index Terms : Satellite Networks, Multiple Access Protocols, Quality of Service.

\section{Introduction}

This paper proposes a modified version of the Packet Reservation Multiple Access scheme with Hindering States (PRMA-HS) 1 for the uplink of Low Earth Orbit - Mobile Satellite Systems (LEO-MSSs) with the SW-CTDMA (Satellite Wideband - Code Time Division Multiple Access) air interface defined in [2, 3. This protocol has been named Code Division-PRMA-HS (CD-PRMA-HS). We assume that a Mobile Terminal (MT) must acquire the reservation of a slot-code per frame to transmit to the satellite. The near-far effect, typical of terrestrial cellular systems, is less evident in the satellite case, since all the users of a cell are about at the same distance from the satellite with about the same Signal-toInterference Ratio (SIR). LEO satellites experience important distance variation due to their high-speed. We will refer to a Globalstar ${ }^{T M}$-like LEO satellite constellation [4] with a minimum elevation angle of $15^{\circ}$ (the maximum Round Trip Delay, RTD, value is $20 \mathrm{~ms}$ ).

Among MTs, we have Voice Terminals (VTs) and Data Terminals (DTs) producing Web surfing traffic. Voice traffic belongs to the conversational class; a VT discards a packet from its buffer if it experiences a transmission delay

E. Gregori et al. (Eds.): NETWORKING 2002, LNCS 2345, pp. 778-789 2002.

(C) Springer-Verlag Berlin Heidelberg 2002 
greater than $D_{\max }$ (=32 ms with standard voice codecs). The packet dropping probability, $P_{d r o p}$, must be lower than or equal to 1\% (Quality of Service, QoS, requirement) for an acceptable voice quality [5]. Data traffic belongs to the background class. The QoS for data transmission is measured by the mean message delay (from the message generation to its complete transmission), $T_{m s g}$.

\section{System Description}

We consider the quasi-synchronous return link envisaged in [3] for the Frequency Division Duplexing (FDD) version of the SW-CTDMA air interface [6]. Frames with length $T_{f}(=20 \mathrm{~ms})$ are divided in $N(=8)$ slots of duration $T_{s}$. On a slot, $N_{C O D E}$ codes $(=8)$ are available for simultaneous transmissions. The elementary resource is a slot-code. We consider bursts (i.e., packets) of one slot [3], 6] and a fixed spreading level for VTs and DTs. With a QPSK modulation, a packet transports 640 bits with a payload $L_{p}=528$ bits [3].

Each VT uses a slow speech activity detector to distinguish between talkspurts (ON state) and silent pauses (OFF state) within a conversation. In the ON state a VT generates one packet per frame [5]. As for a DT producing Web surfing traffic, we refer to the model and related parameter values shown in [7]: a DT alternates between a datagram state (DAT) where messages (i.e., datagrams) are generated and a reading state (READ) where no traffic is produced (see Fig. 1). The number of datagrams generated in the DAT state is geometrically distributed with expected value $m_{N d}=25$. The sojourn time in the READ state and the datagram interarrival time in the DAT state are exponentially distributed with mean values $m_{D p c}=4 \mathrm{~s}$ and $m_{D d}=1 /(2 q) \mathrm{s}, q \in\{1,2,3,4$, $5,6,7\}$, respectively. The $q$ value permits to modulate the DT traffic burstiness. The sojourn time in the DAT state is exponentially distributed, with mean value $m_{L p c}=m_{N d} m_{D d}$. Thus, it is easy to show that the DT traffic source is a 2-state Markov Modulated Poisson Process (2-MMPP).

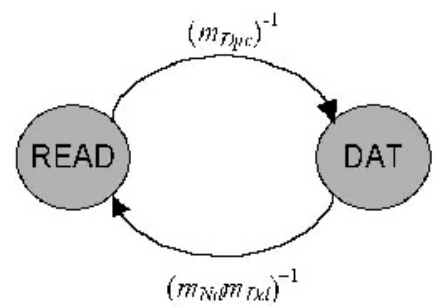

Fig. 1. Adopted model for the DT traffic source.

The mean datagram arrival rate is $\lambda_{d}=m_{L p c} /\left\{m_{D d}\left(m_{L p c}+m_{D p c}\right)\right\}$. Datagrams have a random length in bytes according to the truncated Pareto probability density function shown in [7]. Datagrams are fragmented in packets before transmis- 
sion. According to the packet payload, we have obtained the mean (mean square)

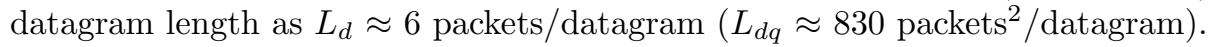

We require $T_{f} \geq R T D_{\max }$, the maximum RTD value experienced by an MT within a cell, so that an MT attempting a transmission on a slot-code receives the outcome before the beginning of the same slot in the next frame. For a conservative study, we consider RTD $\equiv R T D_{\max }$ and $T_{f} \approx n R T D_{\max }$, where $n$ is a divisor of $N$; the slot duration is $T_{s}=T_{f} / N$.

We assume that $M_{v}$ VTs and $M_{d}$ DTs share the use of the same carrier. VTs (DTs) acquire reservations on a talkspurt (datagram) basis. As soon as an MT receives the first packet in its idle buffer, it enters the contending state to acquire a reservation: the MT transmits this packet on available slot-codes, according to its permission probability. Transmission attempts are random in the time-code domain. Orthogonal Variable Spreading Factor (OVSF) codes are used by an MT for making simultaneous attempts (i.e., on the same slot) on different codes. The permission probabilities have been denoted by $p_{v}$ and $p_{d}$ for VTs and DTs, respectively. We have considered $p_{v}>p_{d}$, since VTs have a higher service priority than DTs (see Sections 5 and 7). Simultaneous attempts of different MTs on the same code collide and no reservation is achieved. The satellite sends a reservation notification to the MT, when it successfully decodes its first packet on a slot-code. While waiting for this message, the MT may undertake new random attempts on idle slots. Thus, if the previous attempt has been unsuccessful, this strategy allows a fast re-attempt scheme; otherwise, these further attempts are discarded by the satellite and may hinder the accesses of other MTs. The MT releases the reservation by setting an end-of-transmission flag in the header of the last packet. If a datagram arrives when a DT has already an active transmission with the satellite, the DT maintains the reservation until its buffer is emptied (exhaustive discipline).

OVSF codes are necessary when a multimedia terminal must simultaneously transmit VT and DT traffics. Joint detection is used in uplink, so that the intracell interference has a negligible impact on SIR. Thus, we assume that the system can support $N_{C O D E}$ different MTs transmitting on a given slot with a sufficient quality (outage events are not addressed here).

\section{System Model}

VT and DT behaviors are modeled by two discrete-time Makov chains, where state transitions occur at the slot end. The DT state diagram is shown in Fig. 2 (symbols $A_{d}, U_{d}, \sigma_{d}, \sigma_{d p}$ and $\gamma_{f d}$ of Fig. 2 are defined later). The VT state diagram is characterized by the states enclosed by a box in Fig. 2, where we consider the following probabilities [1]: $\gamma_{v}=1-e^{-T_{s} / t_{1}}, \sigma_{v}=1-e^{-T_{s} / t_{2}}, \gamma_{f v}=$ $1-\left(1-\gamma_{v}\right)^{N}$; finally, the definitions of $A_{v}$ and $U_{v}$ are given below. Starting from the silent pause (SIL), the VT enters the contending (CON) state when a new talkspurt is generated. If the VT makes a successful attempt, the VT enters the block of hindering states from $\operatorname{HIN}(N-1)$ to $\operatorname{HIN}(N-N / n)$. In the $\operatorname{HIN}(N-$ $N / n)$ state the $\mathrm{VT}$ receives the positive acknowledgment from the satellite; then, 


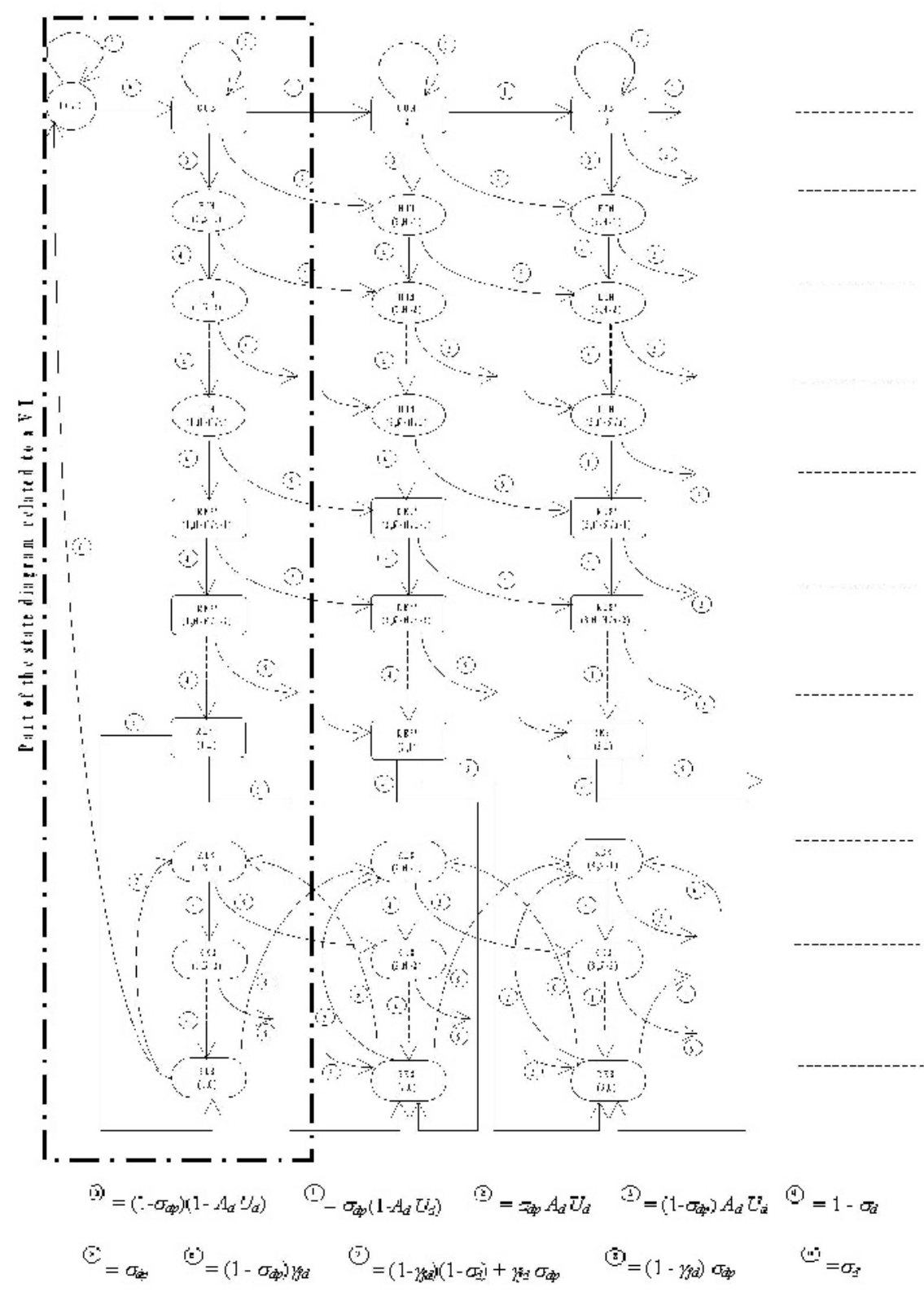

Fig. 2. DT and VT state diagrams.

the VT enters the block from RES' $(N-N / n-1)$ to RES'(1) due to the waiting time before transmitting the next packet on the reserved slot-code. The chain of a DT (Fig. 2) has an infinite number of states (each column of states is for 
a different number of messages in the DT buffer). The $\operatorname{CON}(j)$ state contains all the DTs that need to acquire a reservation with $j$ messages in the buffer. Whereas, $\operatorname{HIN}(j, i), \operatorname{RES}^{\prime}(j, i)$ and $\operatorname{RES}(j, i)$ states are related to the slotcode reserved by the DTs with $j$ datagrams in their buffers.

The system is characterized by the aggregated state $\left\{C_{v}, C_{d}, H_{v}, H_{d}, R_{v}^{*}\right.$, $\left.R_{d}^{*}\right\}$, where $C_{v}\left(C_{d}\right)=$ number of VTs (DTs) in the CON state, $H_{v}\left(H_{d}\right)=$ number of VTs (DTs) in the HIN states, $R_{v}^{*}\left(R_{d}^{*}\right)=$ number of VTs (DTs) that know to have a reservation. Referring to a particular state, we define:

- The probability that a slot-code is not reserved, $P_{f}$ :

$$
P_{f}=1-\left(\frac{R_{v}^{*}+R_{d}^{*}+H_{v}+H_{d}}{N N_{C O D E}}\right) \text {. }
$$

- The probability that a VT (DT) makes a transmission attempt on a slotcode, $A_{v}\left(A_{d}\right)$ :

$$
A_{v}=p_{v} P_{f} \text { and } A_{d}=p_{d} P_{f}
$$

- The probability of a successful transmission attempt for a $\mathrm{VT}, U_{v}$ :

$$
U_{v}= \begin{cases}\left(1-p_{d}\right)^{C_{d}+H_{d}}\left(1-p_{v}\right)^{C_{v}+H_{v}-1} & , \text { if } C_{v} \geq 1, \forall C_{d}, H_{v}, H_{d} \\ 0, & \text { if } C_{v}=0, \forall C_{d}, H_{v}, H_{d}\end{cases}
$$

The corresponding expression for a DT, $U_{d}$, is obtained by changing subscript $v$ with $d$ and vice versa in (3).

A DT in the IDLE state enters the $\mathrm{CON}(1)$ state with probability $\sigma_{d}$, i.e., the probability that the sojourn time in the READ state ends within $T_{s}$; we have:

$$
\sigma_{d}=1-e^{-\frac{T_{s}}{m_{p c}}}
$$

Transitions between parallel columns in the diagram in Fig. 2 are made according to probability $\sigma_{d p}$ (a DT can receive datagrams at any instant in the DAT state):

$$
\sigma_{d p}=\left(1-\frac{1}{N_{D d}}\right)\left(1-e^{-\frac{T_{s}}{m_{D_{d}}}}\right) \quad .
$$

Finally, symbol $\gamma_{f d}$ in Fig. 2 represents the probability that the sojourn time in the DAT state ends in the current frame. In particular, we have: $\gamma_{f d}=$ Prob. \{last datagram of the DAT state $\times$ Prob. \{last packet of the datagram $\}$ :

$$
\gamma_{f d}=\frac{1}{N_{D d}}\left[\frac{k}{\left(L_{d \_ \text {max }}-1\right) L_{p}+1}\right]^{v}
$$

where, according to [7], $k=81.5, v=1.1$ and $L_{d \_\max }=\left\lceil 66666 / L_{p}\right\rceil$, being $\lceil$. the ceiling function. 
Depending on the utilization of slot-codes by VTs and DTs, $\eta_{v}$ and $\eta_{d}$, we obtain the total throughput $\eta_{t o t}$ and the related stability condition as:

$$
\eta_{t o t}=\eta_{v}+\eta_{d}=\frac{\psi_{v} M_{v}\left(1-P_{d r o p}\right)}{N N_{C O D E}}+\frac{\lambda_{d} T_{f} L_{d} M_{d}}{N N_{C O D E}}<1\left[\frac{\text { packets }}{\text { slot code }}\right] .
$$

where $\psi_{v}$ denotes the voice activity factor and the packet dropping probability $P_{\text {drop }}$ will be evaluated in Section 6 .

Since a DT with a reservation uses one slot-code per frame, the following DT buffer stability condition must be fulfilled by the DT traffic load, $\rho_{d}$ :

$$
\rho_{d}=\lambda_{d} T_{s} N L_{d}<1 \quad\left[\frac{\text { packets }}{\text { DT frame }}\right] .
$$

A new Web session is accepted if conditions (7) and (8) are fulfilled (connection admission control).

\section{Equilibrium Point Analysis}

The standard methods for discrete-time Markov-chains cannot be adopted here, since the number of states of the system exponentially increases with the number of MTs. Hence, we have used the Equilibrium Point Analysis (EPA) [5; we study the equilibrium variables (denoted by small letters) by assuming that all the codes of a slot have the same probability to be utilized. Similarly to [1], we obtain the following system in the four unknown terms $c_{v}, c_{d}, h_{v}, h_{d}$ :

$$
\begin{aligned}
& M_{v}=\left[1+\frac{\gamma_{v}}{\sigma_{v}}\right] c_{v}+\left[\frac{1}{\sigma_{v}}+\frac{N}{\gamma_{f v}}\right] N_{C O D E} h_{v} \\
& M_{d}=\frac{c_{d}+\frac{N_{C O D E}}{\sigma_{d}} h_{d}}{1-\lambda_{d} T_{s} L_{d} N} \\
& h_{v}-u_{v}\left(1-\gamma_{v}\right) p_{v} c_{v}\left[1-\frac{h_{v}}{\gamma_{f v}}-\frac{\lambda_{d} T_{s} L_{d}\left(c_{d}+\frac{N_{C O D E}}{\sigma_{d}} h_{d}\right)}{N_{C O D E}\left(1-\lambda_{d} T_{s} L_{d} N\right)}\right]=0 \\
& h_{d}-u_{d} p_{d} c_{d}\left[1-\frac{h_{v}}{\gamma_{f v}}-\frac{\lambda_{d} T_{s} L_{d}\left(c_{d}+\frac{N_{C O D E}}{\sigma_{d}} h_{d}\right)}{N_{C O D E}\left(1-\lambda_{d} T_{s} L_{d} N\right)}\right]=0
\end{aligned}
$$

where $c_{v}\left(c_{d}\right)$ is the equilibrium number of VTs (DTs) in the contending state(s), $h_{v}\left(h_{d}\right)$ is the equilibrium number of VTs (DTs) in each hindering state per code, $u_{v}$ is defined as:

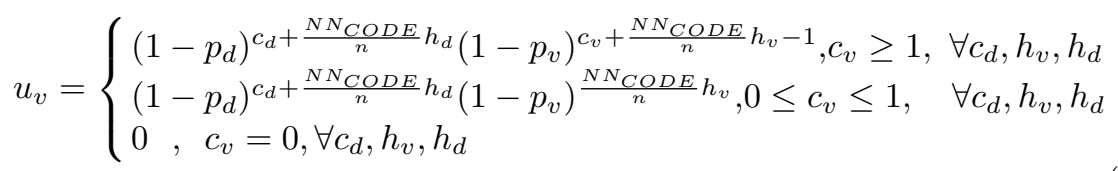

and $u_{d}$ is obtained by changing subscript $v$ with $d$ (and vice versa) in $u_{v}$. 
Since $u_{v}$ and $u_{d}$ have transcendent expressions, we numerically solve the EPA system (91)-12 by using a four-dimensional recursive approach (the obtained solution depends on the starting point, if there are multiple EPA solutions).

\section{Admissible Range for Permission Probabilities}

We need to identify the values of $p_{v}$ and $p_{d}$ that allow a single and stable EPA solution. This problem can be solved by adopting the catastrophe theory that is based on a potential function $V$ defined in the state space $\Omega_{s}=\left\{\left(c_{v}, c_{d}\right) \in \Re \times \Re\right.$ : $0 \leq c_{v} \leq M_{v}$ and $0 \leq c_{d} \leq M_{d}$ and depending on the control space $\Omega_{c}=\left\{\left(p_{v}, p_{d}\right)\right.$ $\left.\in \Re \times \Re: 0<p_{v}, p_{d}<1\right\} V: \Omega_{s} \times \Omega_{c} \rightarrow \Re, V=V\left(\left\{c_{v}, c_{d}\right\},\left\{p_{v}, p_{d}\right\}\right)$. EPA equations (9) and (10) (where $h_{v}=h_{v}\left(\left\{c_{v}, c_{d}\right\},\left\{p_{v}, p_{d}\right\}\right)$ and $h_{d}=h_{d}\left(\left\{c_{v}, c_{d}\right\}\right.$, $\left.\left\{p_{v}, p_{d}\right\}\right)$ are assumed to be implicitly expressed in terms of $c_{v}$ and $c_{d}$ by solving (11) and (12)) are equivalent to the null gradient condition for $V$ with respect to $\Omega_{s},-\nabla_{s} V=0$ :

$$
\left\{\begin{array}{l}
\frac{\partial V}{\partial c_{v}}=\left[1+\frac{\gamma_{v}}{\sigma_{v}}\right] c_{v}+\left[\frac{1}{\sigma_{v}}+\frac{N}{\gamma_{f v}}\right] N_{C O D E} h_{v}\left(\left\{c_{v}, c_{d}\right\},\left\{p_{v}, p_{d}\right\}\right)-M_{v}=0 \\
\frac{\partial V}{\partial c_{d}}=\frac{c_{d}+\frac{N_{C O D E}}{\sigma_{d}} h_{d}\left(\left\{c_{v}, c_{d}\right\},\left\{p_{v}, p_{d}\right\}\right)}{1-\lambda_{d} T_{s} L_{d} N}-M_{d}=0
\end{array} .\right.
$$

The EPA solutions are the critical points of $V, \zeta \in \Omega_{s} \times \Omega_{c}:-\left.\nabla_{s} V\right|_{\zeta}=0$. In [5], for a similar problem, the author proposes the simplifying assumption $c_{d}=M_{d}$. This approach does not allow a correct identification of the region in $\Omega_{c}$ with multiple EPA solutions (i.e., bistable protocol behavior), since the substitution $c_{d}=M_{d}$ strongly modifies the characteristics of the EPA system. Hence, the critical points can be studied by means of the Hessian matrix, $H_{s}(V)[8]$ :

$$
H_{s}(V)=\left(\begin{array}{cc}
\frac{\partial^{2} V}{\partial c_{v}^{2}} & \frac{\partial^{2} V}{\partial c_{d} \partial c_{v}} \\
\frac{\partial^{2} V}{\partial c_{v} \partial c_{d}} & \frac{\partial^{2} V}{\partial c_{d}{ }^{2}}
\end{array}\right)
$$

The signs of the eigenvalues $\varphi_{1}$ and $\varphi_{2}$ of the Hessian matrix in a critical point $\zeta$ identify if $\zeta$ represents a minimum (two positive eigenvalues $\Rightarrow$ stable equilibrium point), a maximum (two negative eigenvalues) or a saddle point (two eigenvalues with different signs). Of course, the correct protocol behavior is allowed only in the region in $\Omega_{c}$ where there is a single solution of (14) that corresponds to a minimum of $V$. If we vary $p_{v}$ and $p_{d}$ in $\Omega_{c}, V$ modifies its shape and, correspondingly, the number of critical points (EPA solutions). Shape changes occur when the determinant of the Hessian matrix vanishes in critical points:

$$
-\left.\nabla_{s} V\right|_{\zeta}=0 \text { and } \operatorname{det}\left\{\left.H_{s}(V)\right|_{\zeta}\right\}=0
$$

For a given $\left(p_{v}, p_{d}\right) \in \Omega_{c}$ we need to evaluate det $\left\{H_{s}(V)\right\}$ in each EPA solution to identify where condition (16]) is fulfilled (= bifurcation set [8]). Unfortunately, this method cannot be practically implemented, since we do not (a priori) know how many solutions the EPA system admits for each $\left(p_{v}, p_{d}\right)$. This is the reason 
why we propose the following heuristic approach. By means of an EPA graphical solution, we have noticed that: $(a)$ the EPA system always admits a solution close to the origin; $(b)$ multiple EPA solutions always entail three EPA solutions; in such cases, the solution with the highest $c_{v}$ and $c_{d}$ values (degenerate solution) is characterized as:

$$
c_{v} \approx c_{v, d e g}=\frac{M_{v}}{\left[1+\frac{\gamma_{v}}{\sigma_{v}}\right]} \quad, \quad c_{d} \approx c_{d, d e g}=M_{d}\left(1-\lambda_{d} T_{s} L_{d} N\right)
$$

Practically, (17) is an EPA solution when, according to (9) and (10) $h_{v} \approx 0$ and $h_{d} \approx 0$. Hence, we use a four-dimensional iterative approach to solve the EPA system for given $M_{v}$ and $M_{d}$ values in two cases: $(i)$ starting point $c_{v}=0, c_{d}$ $=0, h_{v}=0, h_{d}=0$ so as to find the solution closer to the origin; $(i i)$ starting point $c_{v}=c_{v, \text { deg }}, c_{d}=c_{d, \text { deg }}$, considering of course $h_{v}=h_{d}=0$. There are three EPA solutions only when the EPA system admits different solutions in cases $(i)$ and $(i i)$. Accordingly, we scan $\Omega_{c}$ to identify the regions where there are one or three EPA solutions. Thus, in these different cases we may verify (16) to identify the bifurcation set (see below the derivation of the components of the Hessian matrix). Fig. 3 shows the bifurcation set in the control space $\Omega_{c}$ with $q=1, n$ $=1$ for $M_{v}=64 \mathrm{VTs}, M_{d}=40 \mathrm{DTs}$. We note that the region with a single EPA solution obtained here is different from the region identified according to the method proposed in 5 .

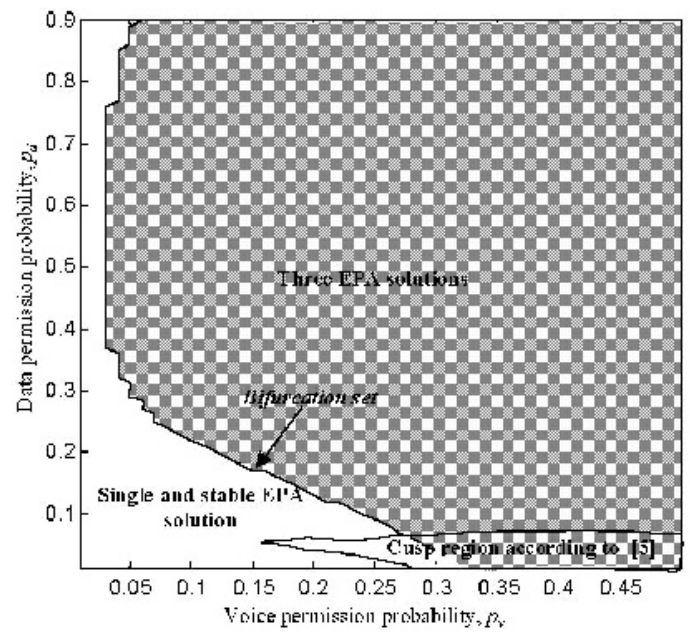

Fig. 3. Bifurcation set in the control space $\Omega_{c}$ for $M_{v}=64$ VTs, $M_{d}=40$ DTs, $q=$ 1 and $n=1$. This figure also contains the cusp region according to [5].

We can characterize the system behavior corresponding to the critical points of the potential function by evaluating the eigenvalues of the Hessian matrix (15), whose entries are obtained as follows: 


$$
\begin{aligned}
& \frac{\partial^{2} V}{\partial c_{v}^{2}}=1+\frac{\gamma_{v}}{\sigma_{v}}+\left[\frac{1}{\sigma_{v}}+\frac{N}{\gamma_{f v}}\right] N_{C O D E} \frac{\partial h_{v}\left(\left\{c_{v}, c_{d}\right\},\left\{p_{v}, p_{d}\right\}\right)}{\partial c_{v}} \\
& \frac{\partial^{2} V}{\partial c_{d}{ }^{2}}=\frac{1+\frac{N_{C O D E}}{\sigma_{d}} \frac{\partial h_{d}\left(\left\{c_{v}, c_{d}\right\},\left\{p_{v}, p_{d}\right\}\right)}{\partial c_{d}}}{1-\lambda_{d} T_{s} L_{d} N} \\
& \frac{\partial^{2} V}{\partial c_{d} \partial c_{v}}=\left[\frac{1}{\sigma_{v}}+\frac{N}{\gamma_{f v}}\right] N_{C O D E} \frac{\partial h_{v}\left(\left\{c_{v}, c_{d}\right\},\left\{p_{v}, p_{d}\right\}\right)}{\partial c_{d}} \\
& \frac{\partial^{2} V}{\partial c_{d} \partial c_{v}}=\frac{\partial^{2} V}{\partial c_{v} \partial c_{d}}(\text { Hessian matrix symmetry condition }) \Rightarrow \\
& \Rightarrow \frac{\partial h_{d}\left(\left\{c_{v}, c_{d}\right\},\left\{p_{v}, p_{d}\right\}\right)}{\partial c_{v}}=\sigma_{d}\left[\frac{1}{\sigma_{v}}+\frac{N}{\gamma_{f v}}\right]\left[1-\lambda_{d} T_{s} L_{d} N\right] \frac{\partial h_{v}\left(\left\{c_{v}, c_{d}\right\},\left\{p_{v}, p_{d}\right\}\right)}{\partial c_{d}}
\end{aligned}
$$

We obtain $\frac{\partial h_{v}}{\partial c_{v}}, \frac{\partial h_{v}}{\partial c_{d}}, \frac{\partial h_{d}}{\partial c_{v}}$, and $\frac{\partial h_{d}}{\partial c_{d}}$ by using the theorem for the derivative of the implicit functions applied to (11) and (12) and by using the symmetry condition given by the last equation in (18). Hence, for each EPA solution $\left(c_{v}, c_{d}\right)$, we solve a system with four transcendent equations in the unknown $\frac{\partial h_{v}}{\partial c_{v}}, \frac{\partial h_{v}}{\partial c_{d}}, \frac{\partial h_{d}}{\partial c_{v}}$, and $\frac{\partial h_{d}}{\partial c_{d}}$ by means an iterative method with starting point $(0,0,0,0)$. Accordingly, we have verified that the EPA solution is always stable in the region in $\Omega_{c}$ where there is a single EPA solution; $p_{v}$ and $p_{d}$ values will be selected in this region, as explained in Section 7.

\section{Performance Analysis}

In order to obtain $P_{d r o p}$ we condition on the aggregated state $\left\{C_{v}, C_{d}, H_{v}, H_{d}\right.$, $\left.R_{v}^{*}, R_{d}^{*}\right\}$. The joint state probability distribution can be derived according to [1] and by considering that there are now $N N_{C O D E}$ resources. $P_{d r o p}$ is obtained on the basis of the method shown in [1], where we have to use a different expression of the conditioned probability that a VT in the CON state has at least one successful attempt on a slot, $P_{s, v}\left(C_{v}, C_{d}, H_{v}, H_{d}, R_{v}^{*}, R_{d}^{*}\right)$ :

$$
P_{s, v}\left(C_{v}, C_{d}, H_{v}, H_{d}, R_{v}^{*}, R_{d}^{*}\right)=1-\left(1-A_{v} U_{v}\right)^{N_{C O D E}} \quad .
$$

The main original aspect for the performance analysis with respect to [1] is given by the derivation of $T_{m s g}$. In particular, $T_{m s g}$ is the sum of two contributions: $(i)$ the mean access delay, $T_{a c c}$, that is the mean time needed by the DT to acquire a reservation (only for a datagram arrived at an empty DT buffer); (ii) the mean transmission delay $T_{\text {delay }}$ due to a queuing system of the $2-M M P P^{[P]} / D / 1$ type (where "2-MMPP ${ }^{[P]}$ " stands for the 2-MMPP arrival process of datagrams of a DT, "D" stands for a deterministic packet transmission time of a frame, "1" means that only one packet can be transmitted per slot).

$$
T_{m s g}=T_{a c c}+T_{\text {delay }} .
$$

$T_{a c c}$ can be approximated 1 as the product of the mean time a DT spends in the contending phase, $E\left[t_{C O N_{d}}\right]$, and the probability that an arriving datagram finds the DT in the IDLE state, $P_{\text {idle }}$ :

\footnotetext{
${ }^{1}$ We have considered here that $T_{a c c}$ is a pure delay contribution; any queuing phenomenon is negligible in the short time spent by a DT in CON states.
} 


$$
T_{a c c}=E\left[t_{C O N_{d}}\right] P_{i d l e}
$$

According to the EPA system, $P_{i d l e}=s_{d} / M_{d}=N_{C O D E} h_{d} /\left(\sigma_{d} M_{d}\right)$. Moreover, $E\left[t_{C O N_{d}}\right]$ can be derived by using the same approach proposed in [1] considering that there are $N N_{C O D E}$ resources. We focus now on $T_{\text {delay }}$. We start by deriving the mean packet delay, $T_{p k t}$. We embed the model to the end of slots and we modify the approximated analytical approach proposed in [9] by taking into account that packets have a compound arrival process due to both the datagram generation process and the variable length of each arrival. The packet arrival process is characterized by the following probability-generating matrix:

$$
\mathbf{Q}(z)=\left[\begin{array}{ll}
p_{11} e^{\lambda_{p} T_{s}[L(z)-1]} & p_{12} \\
p_{21} e^{\lambda_{p} T_{s}[L(z)-1]} & p_{22}
\end{array}\right]
$$

where $\lambda_{p}=2 q$ is the mean datagram arrival rate in the DAT state; $L(z)$ is the probability-generating function of the datagram length distribution in packets; $p_{12}=1-e^{-T_{s} / m_{L p c}}$ is the probability that the source leaves the DAT state in $T_{s} ; p_{11}=1-p_{12} ; p_{21}=1-e^{-T_{s} / m_{D p c}}$ is the probability that the source leaves the READ state in $T_{s} ; p_{22}=1-p_{21}$.

Let $\mathbf{s}=\left(s_{1}, s_{2}\right)^{T}$, where (apex $T$ denotes transposition) $s_{1}$ is the probability of the DAT state and $s_{2}$ is the probability of the READ state.

$$
s_{1}=\frac{p_{21}}{p_{12}+p_{21}} \quad, \quad s_{2}=\frac{p_{12}}{p_{12}+p_{21}} .
$$

Since each source with a reservation transmits one packet per frame, the service time of a packet is equal to $N$ slots. According to [9] and on the basis of (22), the mean packet delay, $T_{p k t}$, results as:

$$
T_{p k t}=N+\frac{\lambda_{1}^{\prime \prime}(1) N^{2}}{2 \rho_{d}\left[1-\rho_{d}\right]}+\frac{N \xi_{1}^{\prime}(1)}{\rho_{d}} \quad[\text { slots }]
$$

where $\lambda_{1}^{\prime \prime}(1)$ and $\xi_{1}^{\prime}(1)$ have quite complex definitions [9]:

$$
\begin{aligned}
& \lambda_{1}^{\prime}(1)=\lambda_{d} T_{s} L_{d} \text { and } \lambda_{2}^{\prime}(1)=p_{11} \lambda_{p} T_{s} L_{d}-\lambda_{1}^{\prime}(1) \\
& \lambda_{1}^{\prime \prime}(1)=\lambda_{1}^{\prime}(1)\left[\frac{L_{d q}-L_{d}}{L_{d}}+\lambda_{p} T_{s} L_{d}+2 \frac{\lambda_{2}^{\prime}(1)}{p_{12}+p_{21}}\right] \\
& \xi_{1}^{\prime}(1) \cong\left\{\mathbf{s}^{T} \mathbf{Q}(0) \mathbf{u}_{1}^{\prime}(1)\right\} /\left\{\mathbf{s}^{T} \mathbf{Q}(0) \mathbf{1}\right\} \\
& \text { being } \quad \mathbf{u}_{1}^{\prime}(1)=\frac{\lambda_{2}^{\prime}(1)}{p_{12}}\left(s_{2},-s_{1}\right)^{T} \quad \text { and } \quad \mathbf{1}=(1,1)^{T}
\end{aligned}
$$

Since $T_{p k t}$ is related to the transmission of a packet in the middle of a datagram, we may consider that $T_{\text {delay }}$ is obtained by adding to $T_{p k t}$ the transmission of the remaining half datagram:

$$
T_{\text {delay }}=T_{p k t}+\frac{L_{d}}{2} N \quad[\text { slots }]
$$




\section{Results}

A CD-PRMA-HS simulator has been realized and very long simulations (about $200 \times 10^{6}$ slots) have been performed to achieve very accurate results. We have carried out a first group of simulation runs for different values of $p_{v}$ and $p_{d}$ in $\Omega_{c}$ in a typical configuration with $M_{v}=64 \mathrm{VTs}, M_{d}=44 \mathrm{DTs}, q=1$ and $n=1$ (these values allow the fulfillment of conditions (77) and (8)). We have obtained that $P_{d r o p}$ has a good behavior for $p_{v}$ around $0.15-0.2$ and $P_{d r o p}$ mildly increases with $p_{d}$. Thus, we have selected $p_{v}=0.2$ and $p_{d}=0.1$. On the basis of Fig. 3, such choice allows a single and stable EPA solution for the typical $M_{v}, M_{d}, q$ and $n$ values considered in this paper. Figs. 4 and 5 compare analytical and simulation results as a function of $q$ for $p_{v}=0.2, p_{d}=0.1, n=$ 1 and considering two cases: $(i) M_{v}=64 \mathrm{VTs}$ and $M_{d}=44 \mathrm{DTs} ;(i i) M_{v}=95$ VTs and $M_{d}=9$ DTs.

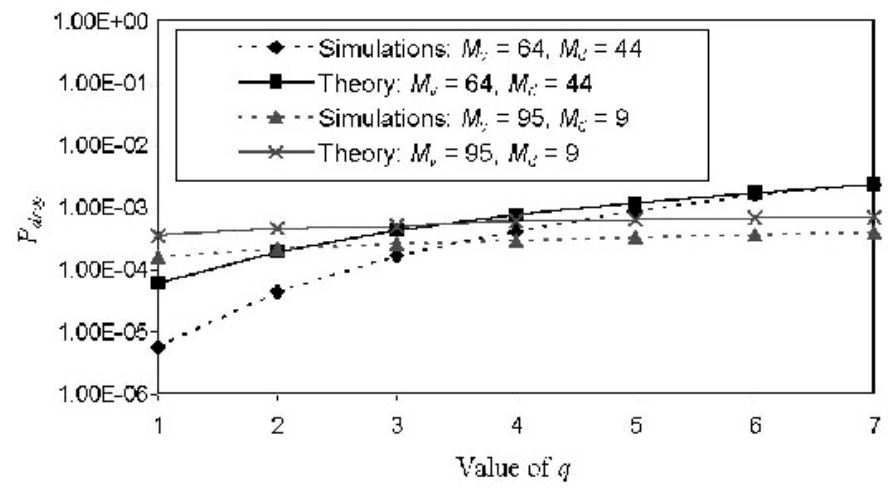

Fig. 4. Comparison of $P_{\text {drop }}$ obtained from theory and simulations.

We note that the $P_{d r o p}$ theory gives a sufficiently close upper bound to simulation results. Moreover, in case $(i)$ the presence of a higher number of DTs entails that the increase of $q$ has a more significant impact on $P_{d r o p}$ than in case $(i i)$. As for $T_{m s g}$, the proposed 2-MMPP analytical approach gives a conservative estimate of the CD-PRMA-HS performance that permits to characterize the service experienced by Web traffic sources. Of course, $T_{m s g}$ increases with $q$. Moreover, $T_{m s g}$ results do not appreciably differ in the two cases, since $T_{m s g}$ is dominated by $T_{\text {delay }}$ that is about the same in the two cases. Let us refer to the maximum number of VTs, $M_{v \max }$, that permits to have $P_{d r o p} \leq 1 \%$, a single and stable solution with $p_{v}=0.2$ and $p_{d}=0.1$; correspondingly, we can consider the total throughput $\eta_{t o t}$. Even considering the very bursty DT traffic case with $q=5$, we have that $M_{v \max }$ is around to $67 \mathrm{VTs}$ for $M_{d}=55 \mathrm{DTs}$ with $n=1$, thus achieving a high $\eta_{t o t}$ value about equal to 0.82 packets/slot-code. 


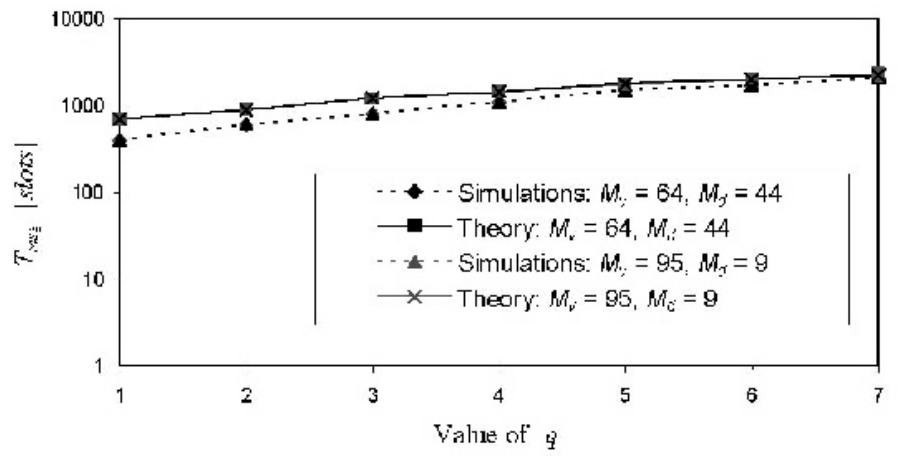

Fig. 5. Comparison of $T_{m s g}$ obtained from theory and simulations.

\section{Conclusions}

The new CD-PRMA-HS protocol has been proposed in this paper for the SWCTDMA air interface in LEO-MSSs, supporting both conversational and background traffics. A stability study and a performance analysis have permitted to characterize the behavior of this new protocol.

\section{References}

1. Benelli, G., Fantacci, R., Giambene, G., Ortolani, C.: Voice and Data Transmissions with a PRMA-like Protocol in High Propagation Delay Cellular Systems. IEEE Trans. on Veh. Tech. 49 (2000) 2126-2147.

2. Taaghol, P., Evans, B. G., Buracchini, E., De Gaudenzi, R., Gallinaro, G., Ho Lee, J., Gu Kang, C.: Satellite UMTS/IMT2000 W-CDMA Air Interfaces. IEEE Comm. Mag. 37 (1999) 116-126.

3. ESA. Wideband Hybrid CDMA/TDMA Option for the Satellite Component of IMT-2000 "SW-CTDMA". ESA Proposal of a Candidate RTT, V1.0, 29 June 1998.

4. Official web site with URL: http://www.globalstar.com.

5. Nanda, S.: Stability Evaluation and Design of the PRMA Joint Voice Data System. IEEE Trans. on Comm. 42 (1994) 2092-2104.

6. ESA. Evaluation Report by European Space Agency IMT-2000 Satellite RTT Evaluation Committee. Sept. 30, 1998.

7. ETSI. Selection Procedures for the Choice of Radio Transmission Technologies of the UMTS (UMTS 30.03 Version 3.1.0). ETSI, Nov. 1997.

8. Saunders. An Introduction to Catastrophe Theory. Cambridge University press, NY, USA, 1980.

9. Steyaert, B., Bruneel, H., Petit, G. H., De Vleeschauwer, D.: A Versatile Queueing Model Applicable in IP Traffic Studies. COST 257 Project, TD (00)-02, Jan. 2000. 